\title{
Forgotten Enemies in the Shadow of COVID-19
}

Parimal Patel' ${ }^{1}$ Khushbu Makadia ${ }^{2}$

${ }^{1}$ Tutor, Community Medicine Department, B. J. Medical College, Ahmedabad, Gujarat, India

${ }^{2}$ Tutor, Community Medicine Department, GMERS Medical College, Himmatnagar, Gujarat, India

Correspondence: Dr. Parimal Patel, Email: drparimalpsm3787@gmail.com

The fight against the corona pandemic is important from the point of its direct impact in terms of high morbidity and moderate mortality but it also could indirectly result in many more victims by disrupting the architecture of our existing health system. So far, It has been seen that due to unprecedented nature of covid19 pandemic and high transmissibility forced the health authorities to give their almost $100 \%$ attention toward covid19 pandemic management and we have either reduced or stopped to pay attention to our known enemies like TB, HIV etc. These are very dangerous infectious diseases that have been known to affect us badly since years with high morbidity and mortality. If we tend to continue forget these known enemies while fighting against the corona, there is a risk of having even greater impact of existing disaster in the coming days.

Official statistics suggest that there were 1.7 million cases of TB in 2017, and it killed 56,277 people, these numbers suggest that TB had a case fatality rate of $3.2 \%$ in 2017 , meaning it was relatively more fatal than covid-19. Where the difference is in the number of cases (multiple times more than covid-19 cases detected so far in the country) and in its treatment (TB has a known cure and treatment regimen unlike covid19).

Table 1: WHO Health Statistics Report Data (INDIA)

\begin{tabular}{|l|l|l|l|}
\hline & \multicolumn{2}{|c|}{ Cause } & $\begin{array}{l}\text { Total Deaths } \\
\text { (Estimated) }\end{array}$ \\
\hline \multicolumn{2}{|c|}{ Communicable, maternal, perinatal and nutritional conditions } & 2490052 \\
\hline A. & Infectious and parasitic diseases & 1199348 \\
\hline & 1. & Tuberculosis & 421437 \\
\hline & 2. & STDs excluding HIV & 3914 \\
\hline & 3. & HIV/AIDS & 62104 \\
\hline & 4. & Diarrhoeal diseases & 409652 \\
\hline & 5. & Childhood-cluster diseases & 38066 \\
\hline & 6. & Meningitis & 34324 \\
\hline & 7. & Encephalitis & 33546 \\
\hline & 8. & Hepatitis & 77304 \\
\hline & & Acute hepatitis A & 2420 \\
\hline & & Acute hepatitis B & 52971 \\
\hline & & Acute hepatitis C & 1081 \\
\hline & & Acute hepatitis E & 20832 \\
\hline & 9. & Parasitic and vector diseases & 63244 \\
\hline 10. & Intestinal nematode infections & 529 \\
\hline & 11. & Leprosy & 7274 \\
\hline 12. & Other infectious diseases & 47954 \\
\hline
\end{tabular}

\begin{tabular}{|l|l|}
\hline \multicolumn{2}{|l|}{ Access this article online: } \\
\hline Quick Response Code & Website: \\
\hline & www.healthlinejournal.org \\
\hline
\end{tabular}

How to cite this article: Patel P, Makadia K. Forgotten Enemies in the shadow of COVID19. Healthline 2020;11(2):125-127. 


\begin{tabular}{|c|c|c|c|c|}
\hline B. & \multicolumn{3}{|c|}{ Respiratory Infectious } & 617245 \\
\hline C. & \multicolumn{3}{|c|}{ Maternal conditions } & 40799 \\
\hline \multirow[t]{5}{*}{ D. } & \multicolumn{3}{|c|}{ Neonatal conditions } & 549737 \\
\hline & \begin{tabular}{l|l}
1. &
\end{tabular} & \multicolumn{2}{|c|}{ Preterm birth complications } & 302277 \\
\hline & 2 & \multicolumn{2}{|c|}{ Birth asphyxia and birth trauma } & 126372 \\
\hline & 3 & \multicolumn{2}{|c|}{ Neonatal sepsis and infections } & 87045 \\
\hline & 4 & \multicolumn{2}{|c|}{ Other neonatal conditions } & 34042 \\
\hline E. & \multicolumn{3}{|c|}{ Nutritional deficiencies } & 82923 \\
\hline \multicolumn{4}{|c|}{ Non-communicable diseases } & 5995333 \\
\hline \multirow{28}{*}{\multicolumn{2}{|c|}{$\begin{array}{l}\text { A.Malignant } \\
\text { neoplasms }\end{array}$}} & \multirow{2}{*}{\multicolumn{2}{|c|}{ 1.Mouth and oropharynx cancers }} & 815148 \\
\hline & & & & 101108 \\
\hline & & a. & Lip and oral cavity & 58983 \\
\hline & & b. & Nasopharynx & 3277 \\
\hline & & c. & Other pharynx & 38848 \\
\hline & & \multicolumn{2}{|l|}{ 2. Oesophagus cancer } & 44432 \\
\hline & & \multicolumn{2}{|l|}{ 3. Stomach cancer } & 62626 \\
\hline & & \multicolumn{2}{|c|}{ 4. Colon and rectum cancers } & 56168 \\
\hline & & \multicolumn{2}{|c|}{ 5.Liver cancer } & 31273 \\
\hline & & \multicolumn{2}{|l|}{ 6.Pancreas cancer } & 16818 \\
\hline & & \multicolumn{2}{|c|}{ 7.Trachea, bronchus, lung cancers } & 89288 \\
\hline & & \multicolumn{2}{|c|}{ 8.Melanoma and other skin cancers } & 4498 \\
\hline & & \multicolumn{2}{|l|}{ 9.Breast cancer } & 80724 \\
\hline & & \multicolumn{2}{|l|}{ 10.Cervix uteri cancer } & 72661 \\
\hline & & \multicolumn{2}{|l|}{ 11.Corpus uteri cancer } & 5308 \\
\hline & & \multicolumn{2}{|l|}{ 12.Ovary cancer } & 22651 \\
\hline & & \multicolumn{2}{|l|}{ 13.Prostate cancer } & 14371 \\
\hline & & 14.Testicular cancer & & 1472 \\
\hline & & 15.Kidney cancer & & 7014 \\
\hline & & 16.Bladder cancer & & 11004 \\
\hline & & 17. Brain and nervous & cers & 17216 \\
\hline & & 18.Gallbladder and b & cancer & 38136 \\
\hline & & 19. Larynx cancer & & 19904 \\
\hline & & 20.Thyroid cancer & & 3673 \\
\hline & & 21.Mesothelioma & & 500 \\
\hline & & 22.Lymphomas, mult & & 30397 \\
\hline & & 23.Leukaemia & & 27864 \\
\hline & & 24.Other malignant $n$ & & 56043 \\
\hline B. & & Other neoplasms & & 14344 \\
\hline C. & & Diabetes mellitus & & 308985 \\
\hline D. & & Endocrine, blood, im & ders & 21130 \\
\hline E. & & Mental and substanc & ders & 31099 \\
\hline F. & & Neurological conditic & & 208999 \\
\hline G. & & Cardiovascular disea & & 2590089 \\
\hline $\mathrm{H}$. & & Respiratory diseases & & 1017179 \\
\hline I. & & Digestive diseases & & 509871 \\
\hline $\mathrm{J}$ & & Genitourinary diseas & & 304702 \\
\hline
\end{tabular}


Healthline Journal Volume 11 Issue 2 (July-December 2020)

\begin{tabular}{|c|c|c|c|}
\hline K & \multicolumn{2}{|c|}{ Skin diseases } & 13236 \\
\hline L. & \multicolumn{2}{|c|}{ Musculoskeletal diseases } & 49210 \\
\hline M. & \multicolumn{2}{|c|}{ Congenital anomalies } & 105119 \\
\hline N. & \multicolumn{2}{|c|}{ Oral conditions } & 479 \\
\hline 0. & \multicolumn{2}{|c|}{ Sudden infant death syndrome } & 5743 \\
\hline \multirow{9}{*}{ A. } & & Injuries & 1083234 \\
\hline & \multicolumn{2}{|c|}{ Unintentional injuries } & 811815 \\
\hline & 1. & Road injury & 299091 \\
\hline & 2. & Poisonings & 31310 \\
\hline & 3. & Falls & 206345 \\
\hline & 4. & Fire, heat and hot substances & 45197 \\
\hline & 5. & Drowning & 63969 \\
\hline & 6. & Exposure to mechanical forces & 18983 \\
\hline & 7. & Other unintentional injuries & 146920 \\
\hline \multirow[t]{4}{*}{ B. } & \multicolumn{2}{|c|}{ Intentional injuries } & 271420 \\
\hline & 1. & Self-harm & 215872 \\
\hline & 2. & Interpersonal violence & 54858 \\
\hline & 3. & Collective violence and legal intervention & 689 \\
\hline
\end{tabular}

Table 2: Comparison of contribution of covid19 with other diseases in terms of deaths due to different group of diseases

\begin{tabular}{|l|l|c|c|c|c|c|}
\hline \multicolumn{1}{|c|}{$\begin{array}{l}\text { Sr. } \\
\text { No. }\end{array}$} & \multicolumn{1}{|c|}{$\begin{array}{c}\text { Total deaths } \\
\text { in year }\end{array}$} & $\begin{array}{c}\text { Midyear } \\
\text { Estimated } \\
\text { Total deaths }\end{array}$ & \multicolumn{3}{|c|}{$\begin{array}{c}\text { Contribution of disease in midyear } \\
\text { total estimated death }\end{array}$} \\
\cline { 5 - 7 } & & & & $\begin{array}{c}\text { Covid19 } \\
(\%)\end{array}$ & $\begin{array}{c}\text { Tuberculosis } \\
\text { (\%) }\end{array}$ & $\begin{array}{c}\text { Mouth and } \\
\text { Oropharynx } \\
\text { cancers (\%) }\end{array}$ \\
\hline 1. & Infectious and parasitic & 1199348 & 599674 & 28084 & $\begin{array}{c}210719 \# \\
(35.14)\end{array}$ & - \\
\hline diseases & Respiratory Infectious & 617245 & 308623 & 9.10 & 68.28 & - \\
\hline 3. & Total deaths & 9568619 & 4784310 & 0.59 & 4.40 & 1.06 \\
\hline
\end{tabular}

Increase in morbidity and mortality due to preventable and treatable diseases may occur if the focus shifts injudiciously from combating these diseases to COVID-19.

In line of above discussion more detailed statistics and inference is explained in the tables given below:

- As on $21^{\text {st }}$ July, 1155191 cases and 28084 deaths due to covid19 have been reported across the country.

- As per the latest available report we can analyze the situation as mentioned in table 2

As we can interpret from these both tables that covid19 as disease is much less lethal than tuberculosis or common cancer like Mouth and oropharynx cancers as overall contribution of covid19 is much less than these two health issues as only $0.6 \%$

of estimated midyear deaths are due to covid19 while due to TB and oral cancer are $4.4 \%$ and $1.1 \%$ respectively.

It doesn't mean that we should relax our all the preventive and curative efforts against covid19 but at the same time other very important and much dangerous and more lethal diseases shouldn't be ignored during this unusual pandemic scenario.

\section{References:}

1. Global Health Estimates 2016: Deaths by Cause, Age, Sex, by Country and by Region, 2000-2016. Geneva, World Health Organization; 2018.

2. Covidindiaupdates.in. 2020. COVID-19 India Updates. [online] Available at: <http://covid indiaupdates.in/> [Accessed 25 July 2020]. 